\title{
An equilibrium study of the liquid-phase sorption of Lead (II) ions on nanoporous carbon materials
}

\author{
A. E. Kucherova, I. V. Burakova, A. E. Burakov, \\ E. V. Galunin, A. V. Babkin, E. A. Neskoromnaya \\ Tambov State Technical University Tambov, Russia \\ Anastasia.90k@mail.ru, iris_tamb68@mail.ru,m-alex1983@yandex.ru, \\ evgeny.galunin@gmail.com, flex_trol@mail.ru, lenok.n1992@mail.ru
}

PACS 81.05.U, 81.07.De, 89.60.Ec

DOI 10.17586/2220-8054-2018-9-1-114-116

\begin{abstract}
The present paper describes the adsorption of lead (II) ions on conventional and nanoporous materials. Equilibrium studies were performed by implementing the empirical Freundlich and Langmuir isotherm models. It was found that all the isotherms constructed on the basis of experimental results fitted well to those models, thereby indicating the efficiency of the nanoporous materials as adsorbents of heavy metals. The experimental lead (II) maximum adsorption capacity of the materials under study - CNTs "Taunit-M", highly porous carbon, CNTs "Taunit", BAU-An activated carbon, and bentonite clay - was found to be 23, 14, 13, 10, and $7 \mathrm{mg} \cdot \mathrm{g}^{-1}$, respectively. Due to the high sorption characteristics and unique physical and chemical properties of these materials, the adsorption technologies developed herein may act as good sustainable options for heavy metal removal from industrial effluents.
\end{abstract}

Keywords: adsorption, heavy metal ions, lead, isotherm, carbon nanotubes.

Received: 19 June 2017

\section{Introduction}

Water pollution by heavy metal ions has become a serious environmental issue especially due to their toxicity and tendency for bioaccumulation [1]. Industrial wastewater is considered the main source of lead impurities. Excessive exposure to lead may result in anemia, mental retardation, coma, seizures, and bizarre behavior. Thus, it is necessary to remove this species from wastewater prior to its discharge into the environment. There exist various chemical and physical-chemical methods for the treatment of lead-containing wastewater: chemical precipitation, electrochemical reduction, ion exchange, biosorption, adsorption, among others [2]. Even though there are so many adsorbents available, there is still a need for developing novel materials to more effectively remove metal ions [3]. In this regard, a new type of adsorbent materials has been proposed - nanoporous carbon. It retains a great potential for the removal of a wide range of pollutants such as lead, chromium, nickel, copper, and other ions [4]. Researchers have found that nanoporous carbon materials such as carbon nanotubes (CNTs) have a relatively large surface area, extraordinary surface morphology and good chemical and mechanical properties that provide a good opportunity for the removal of heavy metals [5].

\section{Experimental}

\subsection{Research methods}

Experimental studies on the lead (II) adsorption from aqueous solutions were performed with conventional and nanoporous materials (see Sec. 2.2 below). Each series consisted of six 50-mL tubes containing the corresponding adsorbent. $15 \mathrm{~mL}$ of $200,500,700,1000$ and $2400 \mathrm{mg} \cdot \mathrm{L}^{-1} \mathrm{~Pb}\left(\mathrm{NO}_{3}\right)_{2}$ solutions were separately added into those tubes using Pasteur pipettes. Then, the tubes were end-over-end shaken on Bio RS-24 programmable rotators at $100 \mathrm{rpm}$ during the equilibrium time previously found for the adsorbent. After the adsorption process was finished, the solutions were centrifuged using a $5810 \mathrm{R}$ centrifuge at $10,000 \mathrm{rpm}$ for $10 \mathrm{~min}$ to separate the phases. Next, the liquid phase was placed into $30-\mathrm{mL}$ tubes with the Pasteur pipettes, preventing ingress of the solid phase and the suspension thereof. Finally, the metal concentrations in the solutions were measured according to the method of quantitative elemental analysis on an MGA-915MD atomic absorption spectrometer. 


\subsection{Adsorbents}

In the present research, the following nanoporous adsorption materials were employed: "Taunit"-series CNTs (Taunit, Taunit-M) produced by JSC "NanoTechCenter" (Tambov, Russia), and highly porous carbon (HPC) also synthesized at JSC "NanoTechCenter" via alkaline activation of a carbon product obtained by heat treatment of a mixture of phenol-formaldehyde resin, carboxymethyl cellulose and graphene nanoplatelets (this procedure may considerably increase the quality of purification of aqueous systems containing heavy metals, in particular, lead.

Besides, the efficiency of the above-mentioned nanomaterials was estimated in comparison with the following conventional adsorbents used as references: BAU-A activated carbon produced by JSC "Sorbent" (Perm, Russia), and bentonite clay (BC) acquired from "Bentonit Company" (Moscow, Russia).

\section{Adsorption studies}

The adsorption capacity is an important factor since it determines how much of an adsorbent is required for quantitative enrichment of adsorbates from a given solution. In the present research to study lead (II) adsorption on conventional and nanoporous materials, the adsorption equilibrium data were fitted using the Langmuir and Freundlich models, which correspond to homogenous and heterogeneous adsorbent surfaces, respectively.

The Langmuir model assumes that adsorption takes place at uniform energy sites on the adsorbent surface, whereas the Freundlich model is an empirical equation that considers multilayer adsorption due to the diversity of adsorption sites. The linear Langmuir and Freundlich isotherm models can be expressed as given in Eqs. (1) and (2):

$$
\begin{gathered}
\frac{C_{e}}{q_{e}}=\frac{1}{b q_{m}}+\frac{C_{e}}{q_{m}}, \\
\lg q_{e}=\lg K+\frac{1}{n} \lg C_{e},
\end{gathered}
$$

where, $C_{e}\left(\mathrm{mg} \cdot \mathrm{L}^{-1}\right)$ is the equilibrium concentration of lead (II) in the solution, $q_{e}\left(\mathrm{mg} \cdot \mathrm{g}^{-1}\right)$ is the equilibrium adsorption capacity of the material, $q_{m}\left(\mathrm{mg} \mathrm{g}^{-1}\right)$ is the maximum amount of lead (II) adsorbed per unit mass of the adsorbent required for monolayer coverage of the surface, $b\left(\mathrm{~L} \cdot \mathrm{mg}^{-1}\right)$ is a constant related to the adsorption free energy, $K$ and $n$ are Freundlich constants $\left(K\left(\mathrm{~L} \cdot \mathrm{g}^{-1}\right)\right.$ represents multilayer adsorption capacity at unit concentration of adsorbate and is a relative measure of adsorption capacity of adsorbents, and $n$ represents adsorption intensity which varies with heterogeneity of the adsorbent surface - when $n$ approaches to zero, the surface site heterogeneity increases; for a favorable adsorption process, $n$ should lie in the range 1-10 [6]).

The adsorption parameters found using the above-mentioned models are presented in Table 1.

TABLE 1. Isotherm constants obtained for the Lead (II) adsorption on the conventional and nanoporous materials

\begin{tabular}{|c|c|c|c|c|c|c|}
\hline \multirow{2}{*}{ Material } & \multicolumn{3}{|c|}{ Langmuir constants } & \multicolumn{2}{c|}{ Freundlich constants } \\
\cline { 2 - 7 } & $q_{m}$ & $b$ & $R^{2}$ & $K$ & $n$ & $R^{2}$ \\
\hline BAU-A & 83.33 & $5.19 \cdot 10^{-5}$ & 1.000 & 0.0005 & 1.048 & 0.999 \\
\hline BC & 10.20 & $4.94 \cdot 10^{-4}$ & 0.989 & 0.017 & 1.323 & 0.985 \\
\hline HPC & 23.26 & $9.06 \cdot 10^{-4}$ & 0.993 & 0.182 & 1.736 & 0.900 \\
\hline Taunit & 27.74 & $6.66 \cdot 10^{-4}$ & 0.982 & 0.148 & 1.678 & 0.902 \\
\hline Taunit-M & 62.50 & $3.93 \cdot 10^{-4}$ & 0.988 & 0.175 & 1.546 & 0.905 \\
\hline
\end{tabular}

It was found that for a range of small lead (II) concentrations $\left(200-700 \mathrm{mg} \cdot \mathrm{L}^{-1}\right)$, it is preferable to implement the Langmuir model. Based on the obtained values of the sorption equilibrium constants of this equation, it can be assumed that the lead ions in lower concentrations interact with active sorption sites located at the surface of the materials under study and are responsible for physical adsorption. The range of high concentrations does not satisfy the boundary conditions for the applicability of the Langmuir model with the monomolecular sorption mechanism, and active sorption within this range can serve as the foundation for an indirect confirmation of the applicability of the Freundlich model. Based on the values of the empirical constants of the Freundlich equation, favorable conditions for chemical adsorption can be assumed. This sorption type is associated with energy heterogeneity of the adsorption sites. 
Furthermore, the obtained experimental results demonstrate that the lead (II) sorption capacity increases in the following sequence: BC $\left(7 \mathrm{mg} \cdot \mathrm{g}^{-1}\right)<$ BAU-A $\left(10 \mathrm{mg} \cdot \mathrm{g}^{-1}\right)<$ Taunit $\left(13 \mathrm{mg} \cdot \mathrm{g}^{-1}\right)<\mathrm{HPC}\left(14 \mathrm{mg} \cdot \mathrm{g}^{-1}\right)<$ Taunit-M (23 $\left.\mathrm{mg} \cdot \mathrm{g}^{-1}\right)$.

Thus, it can be concluded that the nanoporous carbon materials can make a significant contribution to the adsorption process and can be successfully used to remove heavy metals (in particular, lead) from water and wastewater in contrast to the materials (activated carbons and clays) commonly used in the industry.

\section{Acknowledgements}

The research was funded by the Ministry of Education and Science of the Russian Federation (Project No. 16.1384.2017/PCh).

\section{References}

[1] Heidari A., Younesi H., Mehraban Z. Removal of Ni(II), Cd(II), and $\mathrm{Pb}(\mathrm{II})$ from a ternary aqueous solution by amino functionalized mesoporous and nano mesoporous silica. Chem. Engin. J., 2009, 153(1-3), P. 70-79.

[2] Saeed A., Iqbal M., Akhtar M.W. Removal and recovery of lead(II) from single and multimetal (Cd, Cu, Ni, Zn) solutions by crop milling waste (black gram husk). J. Hazard. Mater, 2005, 117, P. 65-73.

[3] Hsieh S.H. Growth of carbon nanotube on micro-sized $\mathrm{Al}_{2} \mathrm{O}_{3}$ particle and its application to adsorption of metal ions. J. Mater. Res., 2006, 21, P. 1269-1273.

[4] Tehrani M.S., Azar P.A., Namin P.E., Dehaghi S.M. Removal of lead ions from aqueous solution using multi-walled carbon nanotubes: The effect of functionalization. J. Appl. Environ. Biol. Sci., 2014, 4, P. 316-326.

[5] Yang S., Hu J., Chen C., Shao D., Wang X. Mutual effects of $\mathrm{Pb}(\mathrm{II})$ and humic acid adsorption on multiwalled carbon nanotubes/polyacrylamide composites from aqueous solutions. Environ. Sci. Technol., 2011, 45, P. 3621-3627.

[6] Ghaedi M., Khafri H.Z., Asfaram A., Goudarzi A. Response surface methodology approach for optimization of adsorption of Janus Green B from aqueous solution onto $\mathrm{ZnO} / \mathrm{Zn}(\mathrm{OH})_{2}-\mathrm{NP}-\mathrm{AC}$ : kinetic and isotherm study. Spectrochim. Acta Mol. Biomol. Spectrosc., 2016, 152, P. 233-240. 